УДК 616.89-008.454:615.86:159.938.362.33

Для цитирования: Бахмутский И.Н., Косенко В.Г., Шулькин Л.М., Губа С.А., Бахмутский Н.Г. Повторяющаяся транскраниальная магнитная стимуляция в лечении депрессии (обзор литературы). Сибирский вестник психиатрии и наркологии. 2019; 1 (102): 87-98. https://doi.org/10.26617/1810-3111-2019-1(102)-87-98

\title{
Повторяющаяся транскраниальная магнитная стимуляция в лечении депрессии (обзор литературы)
}

Бахмутский И.Н. ${ }^{1,2}$, Косенко В.Г. ${ }^{1}$, Шулькин Л.М. ${ }^{1}$, Губа С.А. ${ }^{2}$, Бахмутский Н.Г. ${ }^{1}$

${ }^{1}$ Кубанский государственный медицинский университет

Россия, 350063, Краснодар, ул. Седина, 4

${ }^{2}$ Специализированная клиническая психиатрическая больница № 1

Россия, 350000, Краснодар, ул. Красная, 1

\section{PEЗЮME}

Повторяющаяся транскраниальная магнитная стимуляция (пТМС) является эффективным методом лечения депрессии. В многочисленных исследованиях представлены доказательства того, что пТМС действительно считается многообещающим методом лечения, однако её эффективность зачастую оказывается скромной. В своей работе мы рассматриваем эти доказательства и обсуждаем несколько причин, которые могут объяснить скромную терапевтическую эффективность в большинстве из них. В том числе анализируется наиболее частое использование только левосторонней стимуляции дорсолатеральной префронтальной коры (DLPFC), которое не может быть оптимальным при сравнении с двусторонней стимуляцией. Обсуждается применение субоптимальных методов для нацеливания на DLPFC (т.е. используемого 5-сантиметрового переднего метода), тем самым ограничивая потенциал терапии только целевой формой лечения. Короткая (от 2 до 4 недель) продолжительность терапии и интенсивность стимуляции при проведении процедуры может быть недостаточной, если не учитывать расстояние между катушкой и корой, от которого зависит пТМС-индуцированный антидепрессивный ответ. Будущие исследования, направленные на устранение вышеупомянутых ограничений, необходимы для потенциальной оптимизации эффективности этого многообещающего варианта лечения депрессии, особенно её фармакорезистентных форм. Также важно, чтобы работы были ориентированы на исследование механизмов терапевтической эффективности пТМС, так как их понимание может быть преобразовано в успешное лечение. По многим причинам, которые будут рассмотрены, особенно важным механизмом, связанным с терапевтической эффективностью при лечении депрессии, может являться возбудимость коры головного мозга.

Ключевые слова: депрессия, повторяющаяся транскраниальная магнитная стимуляция, кора головного
мозга.

\section{ВВЕДЕНИЕ}

По данным ВОЗ, депрессия - один из основных факторов риска для инвалидности больных. Депрессивное изменение настроения, наряду с искажением когнитивных процессов, сопровождается моторным торможением, снижением побуждений к деятельности, соматовегетативными дисфункциями. Депрессивная симптоматика негативно влияет на социальную адаптацию и качество жизни $[1,2]$. Хотя существуют эффективные методы лечения депрессии, только 30-40\% больных полностью освобождаются от её симптомов при использовании антидепрессантов. Эффективность антидепрессантов (как классических, так и с селективным механизмом действия) не превышает 60\% [3]. Эти статистические данные подчеркивают необходимость поиска альтернативных стратегий лечения для оптимизации его результатов, однако доступных альтернативных способов терапии депрессии и её рецидивов мало.
Одной из таких альтернатив является электросудорожная терапия (ЭСТ), однако эта методика лечения связана со значительными побочными эффектами, в первую очередь с ухудшением памяти пациентов после процедур. Также ЭСТ индуцирует припадок, требует использования общей анестезии, тем самым делает эту процедуру инвазивной с повышенным риском осложнений. Кроме того, стигма, связанная с ЭСТ, часто ограничивает ее широкое признание в качестве лечения симптомов депрессивного расстройства $[4,5]$.

Транскраниальная магнитная стимуляция (TMC) - уникальная экспериментальная методика, позволяющая исследователям неинвазивно изучать кору головного мозга у здоровых и больных пациентов. Используется в качестве инструмента исследования при измерении различных корковых явлений, таких как корковое торможение и корковая пластичность, а также для изучения когнитивных механизмов [6]. 
В ряде работ показано, что ТМС является методикой для лечения таких заболеваний, как депрессия и шизофрения [7, 8, 9]. Ещё в 1831 г. Майкл Фарадей продемонстрировал, что ток можно навести во вторичной цепи, когда её размещают рядом с первичной, по которой протекает изменяющийся во времени ток. Изменяющееся электрическое поле создает магнитное, которое в соответствии с законом Фарадея вызывает ток в соседней проводящей системе. При ТМС электрический заряд накапливается в конденсаторах. Периодическая разрядка этой энергии от конденсатора и через проводящую катушку формирует изменяющееся во времени электрическое поле. Это электрическое поле создает магнитное, которое вызывает ток во вторичном проводящем материале, таком как нейроны. Разряды ТМС над кожей головы вызывают деполяризацию проводящей нервной ткани, расположенной непосредственно под катушкой. Так как промежуточная ткань между катушкой и корой (скальп и череп) в значительной степени непроводящая, магнитное поле проникает через эти ткани практически без ослабления. Ориентация и интенсивность тока, проходящего через катушку, определяет тип стимулируемой ткани, а также силу этой стимуляции.

Таким образом, небольшими катушками, напоминающими по форме цифру 8, активируются нейроны в области коры головного мозга площадью около $3 \mathrm{~cm}^{2}$, а магнитное поле распространяется на глубину примерно 2 см [10]. В большинстве исследований 8-образные катушки удерживались над корой ровно и примерно под углом $45^{\circ}$ от срединного положения, перпендикулярно к центральной борозде. Это индуцирует ток от заднего к переднему направлению, перпендикулярно к нисходящим пирамидальным нейронам и параллельно к интернейронам, которые модулируют пирамидальное клеточное зажигание (cell-firing) [11]. Ориентация между катушкой и нижележащей нервной тканью позволяет исследователям избирательно активировать различные группы нейронов, что, по всей видимости, является ключом к пониманию механизмов терапевтической эффективности ТМС. Следовательно, в силу того факта, что ТМС активирует нейроны транссинаптически [12] (т.е. через активацию интернейронов), нейронная стимуляция может избирательно активировать или ингибировать кору, что может служить индикатором к лечебным эффектам при воздействии.
Повторяющаяся транскраниальная магнитная стимуляция оказалась эффективным терапевтическим средством для лечения ряда психоневрологических расстройств, включая депрессию, шизофрению, эпилепсию и т.д. [13, $14,15]$.

\section{ЦЕЛЬ ИССЛЕДОВАНИЯ}

Провести анализ литературы, оценивающей эффективность пТМС в лечении депрессии.

При изучении литературы внимание акцентировано на понимание терапевтического влияния пТМС на симптоматику депрессии с помощью нейрофизиологических механизмов. В заключение мы отметили важные ограничения использования на сегодняшний день этой методики лечения и потенциальные направления для будущих исследований, пытающихся оптимизировать антидепрессивную эффективность пТМС при депрессивном расстройстве.

\section{РЕЗУЛЬТАТЫ И ОБСУЖДЕНИЕ}

пТМС включает стимуляцию коры головного мозга чередой магнитных импульсов на частотаx от 1 до 50 Гц, в отличие от одноимпульсной $\mathrm{TMC}$, в которой частота стимуляции меньше 1 Гц [16]. Низкочастотная (Low-Frequency Repetitive Transcranial Magnetic Stimulation, LFR) пТМС (около 1 Гц) может ингибировать кортикальную активность и поэтому используется в лечении симптомов, связанных с чрезмерной активацией коры головного мозга.

J. Chen, C. Zhou, B. Wu [17] продемонстрировали, что низкочастотная стимуляция в течение 15 минут вызывает временное торможение коры. Другими авторами, напротив, показано, что высокочастотная стимуляция повышает возбудимость коры [18]. Исследования нейрофизиологических эффектов разных частот пТМС (т.е. 1, праймирование [от 6 Гц до 1 Гц], 10 или 20 Гц) для ингибирования коры головного мозга у 12 здоровых испытуемых [19] указали на значительное подавление механизмов, связанных с GABAB-опосредованным рецептором, ингибирующим нейротрансмиссию более высокими частотами стимуляции (10 и 20 Гц), что более эффективно по сравнению с низкими частотами стимуляции (1 Гц). Таким образом, эти выводы показывают, что некоторые терапевтические эффекты пТМС могут быть скоординированы усиленным образованием $\gamma$ аминомасляной кислоты (GABA), что опосредует подавление нейротрансмиссии. Это согласуется с выводом о том, что GABАергическая нейротрансмиссия нарушается при депрессии [20] и усиливается ЭСТ или лечением ингибиторами обратного захвата серотонина [21]. 
Первые опубликованные исследования указывали на то, что пТМС эффективна для улучшения настроения у пациентов с депрессивным расстройством [22]. Впоследствии в многочисленных изданных работах была дана оценка эффективности пТМС при лечении депрессии [23].

Исследования пТМС высокой частоты (от 10 до 20 Гц) над левой дорсолатеральной префронтальной корой (DLPFC) продемонстрировали эффективность в лечении депрессии [24, 25]. M.S. George, E.M. Wassermann, W.A. Williams et al. [26] первыми сообщили о скромном улучшении симптоматики у 6 пациентов с резистентной к лечебным препаратам депрессией в открытом исследовании с использованием пТМC (средний балл по шкале Hamilton Depression Rating Scale (HDRS) снизился с 23,8 до 17,5). Затем A. Pascual-Leone, B. Rubio, F. Pallardo et al. [27] показали, что пТМС эффективна при лечении депрессивных симптомов у 17 пациентов с лекарственно-устойчивой депрессией с психотическими особенностями при ежедневном применении в течение одной недели. Это было уже рандомизированное плацебо контролируемое исследование с множественным пересечением, с фиктивной пТМС и стимуляцией различных областей коры, используемой в качестве контроля. Недавно другими исследователями было сообщено о значительном улучшении симптомов в группе пациентов с тяжёлым депрессивным расстройством и терапевтически резистентной депрессией в двухнедельных плацебо контролируемых перекрестных исследованиях реальной и фиктивной высокочастотной (High-Frequency Repetitive Transcranial Magnetic Stimulation, HFL) пTMC [28, 25]. Хотя пТМС может быть альтернативой электросудорожной терапии при лечении депрессии, несколько исследований, оценивающих их эффективность и приемлемость, дали противоречивые результаты. Как упоминалось выше, метаанализ показал, что эффективность пТМС связана с параметрами стимула. Другие исследования в рамках метаанализа за последнее десятилетие сообщили, что ЭСТ более эффективна, чем пТМС, для лечения большого депрессивного расстройства. пTMS была столь же эффективна, как и ЭСТ при непсихотической депрессии, но ЭСТ была более эффективной для пациентов с психотическими особенностями. Однако доказательств относительно долгосрочной или среднесрочной эффективности пТМС все еще недостаточно [29].
Несколько исследований продемонстрировали использование LFR пTMC ( $\leq 1$ Гц) над правой DLPFC с оценкой эффективности при депрессии. В большом двойном слепом исследовании у 70 пациентов с депрессией E. Klein, I. Kreinin, A. Chistyakov et al. [30] изучили терапевтическую эффективность пТМС (частота 1 ГЦ) над правой DLPFC. Пациенты были случайным образом разделены на группы со стимуляцией пТМС и плацебо. После 2 недель лечения 49\% пациентов, получавших пТМС, были классифицированы как респондеры (снижение более чем на $50 \%$ баллов по шкале HDRS), тогда как только $25 \%$ пациентов были респондерами в группе с плацебо. Другие, подобные этому исследования также подтвердили эффективность пТМС с такими же параметрами при лечении депрессии [31, 32].

Но есть исследования, которые были двусмысленными или показали недостаточную эффективность пТМС при лечении депрессии. R.M. Berman, M. Narasimhan, G. Sanacora et al. [33] в двойном слепом плацебо контролируемом исследовании сообщили только о незначительном снижении симптомов депрессии после 10-дневного курса HFL пТМС при воздействии на левую DLPFC. C. Loo, P. Mitchell, P. Sachdev et al. [34] не выявили существенной разницы при использовании высокочастотной $\mathrm{HFL}$ пTMC на левую DLPFC в сравнении с плацебо у 18 пациентов с депрессией после 2 недель лечения. Можно объяснить эти противоречивые результаты следующим образом. Вопервых, большинство пациентов, включенных в эти исследования, были устойчивы к лечению антидепрессантами и представляли собой относительно гетерогенную группу, основное расстройство сочеталось у них с другими сопутствующими заболеваниями. Во-вторых, параметры стимуляции (частота, интенсивность и продолжительность воздействия) варьировались в исследовании с целью отработки оптимальных параметров для терапевтического ответа. В-третьих, одновременный прием лекарств в этих исследованиях не вносил ясность в проблему, улучшились ли симптомы депрессии за счёт одной стимуляции пТМС, одного фармпрепарата или комбинации того и другого. В-четвертых, не было стойкого метода для точной локализации префронтальной коры головного мозга при использовании пТМС, поэтому могли стимулироваться другие области коры у разных пациентов, что возможно повлияло на результаты лечения. 
T.A. Kimbrell, J.T. Little, R.T. Dunn et al. [35] попытались уточнить некоторые из этих факторов, рассмотрев возможность ответа подгруппы пациентов с депрессией, сопровождающейся церебральным гипометаболизмом, на HFL пТМС, а пациентов с церебральным гиперметаболизмом на LFR пTMC. Таким образом, они попытались обосновать эти ответы на стимуляцию механизмами церебральной патофизиологии. В этом 2-недельном рандомизированном перекрестном исследовании участвовали 13 субъектов, церебральный метаболизм оценивался отсканированными изображениями позитронно-эмиссионной томографии с применением фтордезоксиглюкозы F 18. Обнаружено, что пациенты с исходным уровнем гипометаболизма лучше реагировали на HFL пTMC в сравнении с пациентами с исходным уровнем гиперметаболизма, имевшими тенденцию к лучшему ответу на LFR пTMC. В совокупности эти исследования обеспечили многообещающие доказательства того, что пТМС представляет собой потенциально новый метод лечения депрессии.

В большинстве исследований по изучению терапевтического эффекта пТМС, описанных выше, обычно использовали 2-недельную продолжительность лечения. Обоснование увеличения продолжительности терапии кажется очевидным, если учесть, что 2 недели ЭСТ часто недостаточны при резистентной депрессии и лечение часто продлевают до 5 недель. В настоящее время ведутся работы с использованием 20 и более процедур для оптимизации клинической эффективности пТМС. P.B. Fitzgerald, K.E. Hoy, R.J. Anderson, Z.J. Daskalakis [36] рандомизировали 60 пациентов в группы по 20 человек в каждой с HFL пTMC (10 Гц), LFR пTMC (1 Гц) или фиктивной стимуляцией. У всех пациентов были резистентные к фармпрепаратам депрессии или неудачные результаты после лечения антидепрессантами. В трёх исследуемых группах не было основных клинических или демографических различий. В двойной слепой фазе исследования отмечен явный антидепрессивный эффект в обеих активных группах, который был лучше, чем ответ на фиктивную стимуляцию. Симптомы депрессии продолжали улучшаться в обеих активных группах и через 4 недели наблюдения. После 4 недель лечения среднее изменение показателей шкалы MontgomeryAshberg Depression Rating Scale (MADRS) от исходного уровня составило $48,0 \%$, среднеквадратичное отклонение $17,9 \%$ (95\% ДИ, от $15,1 \%$ до $87,5 \%$ ).
Эти результаты показывают, что как HFL, так и LFR пTMC обладают значительной терапевтической эффективностью. Для клинического ответа, по-видимому, требуется не менее 20 сеансов лечения с предложенными параметрами. D.H. Avery, P.E. Holtzheimer, W. Fawaz et al. [37] сравнили 3-недельную HFL пTMC с фиктивной пТМС у 68 пациентов с резистентной депрессией и продемонстрировали, что $30,6 \%$ больных, получавших HFL пTMC, соответствовали критериям терапевтического ответа (снижение симптомов более чем на $50 \%$ по шкале HDRS). Это было статистически лучше $(\mathrm{p}<0,05)$ фиктивной стимуляции, при которой только $6,1 \%$ пациентов соответствовали критериям терапевтического ответа. Кроме того, уровень ремиссии для HFL пTMC группы был равен $20 \%$ (HDRS менее 8), что достоверно выше по сравнению с $3 \%$ в фиктивной группе.

Наиболее достоверной на сегодняшний день является работа, оценивающая эффективность HFL пTMC в сравнении с фиктивной пTMS, проведенная частным производителем приборов для TMC (Neuronetics Pty Ltd), которая включила рандомизированное исследование использования HFL пTMC (10Гц) по сравнению с плацебо у 301 пациента без лечения фармпрепаратами и не получавших предшествующей терапии антидепрессантами [38]. Результаты этого исследования показали, что после 6 недель HFL пTMC было отмечено снижение на 6,3 балла по 24-балльной HDRS относительно фиктивной пТМС и разница в 3,4 балла относительно ложной стимуляции отмечена по 17-балльной HDRS. Наконец, 24\% пациентов, получающих HFL пTMC, соответствовали критериям ответа на лечение, по сравнению с $12 \%$ больных, получающих плацебо.

Несколько новых подходов магнитной стимуляции были исследованы в попытке улучшить эффективность лечения пТМС, используя двустороннюю стимуляцию. Такие исследования были инициированы, в частности, как результат неопровержимого доказательства того, что двусторонняя ЭСТ превосходит одностороннюю ЭСТ при аналогичных параметрах стимула и из свидетельств того, что как HFL, так и LFR пTMS приводят к антидепрессивному ответу. Первоначальная попытка одновременного применения двусторонней высокочастотной пТМС была безуспешной [39]. Впоследствии были проведены многочисленные испытания последовательной двусторонней пTMC, сочетающей HFL и LFR пTMC. 
В небольшой серии случаев с 4 пациентами из 7, классифицированными как отвечающие на двустороннюю стимуляцию [40] и в 5-дневном исследовании лечения, сравнивающего HFL пТМС с двусторонним пТМС и воздействием на левое DLPFC как HFL, так и LFR пTMC (10 Гц и 1 Гц), не выявили различий между группами [41]. Однако последнее исследование было относительно коротким (5 дней подряд) и включало небольшую выборку $(\mathrm{n}=12)$. Таким образом, неспособность показать групповые различия была, вероятно, вторичной по отношению к высоким показателям ответа, продемонстрированными всеми группами лечения. В свою очередь, это также можно объяснить отсутствием группы плацебо.

В исследовании пТМC (n=38) A. Hausmann, G. Kemmler, M. Walpoth et al. [42] не сообщили о значимой разнице в эффекте лечения между двусторонней пTMC и HFL пTMC. Это исследование ставило в тупик тем, что в ходе лечения пТМС использовались антидепрессанты. M. Rybak, R. Bruno, L. Turnier et al. [43] изучали эффективность двусторонней пТМС и $\mathrm{HFL}$ пTMS у 18 пациентов с резистентной депрессией. Это исследование также не показало значительных различий в эффекте между группами активного лечения. Однако число больных было недостаточным, чтобы показать межгрупповые различия; 6 из 9 пациентов в группе с двусторонней пТМС и 5 из 9 пациентов в группе HFL-пTMC соответствовали критериям ответа на лечение. Этот высокий коэффициент ответа может отражать отсутствие плацебо контроля.

Тем не менее эти исследования ограничены в нескольких направлениях. Двусторонняя пТМС не сравнивалась с односторонней и фиктивной пТМС в достаточно большой выборке пациентов $\quad(\mathrm{n}=50)$, чтобы минимизировать ошибку и стабилизировать оценку статистических параметров. Ни одно из исследований не проводилось более 10 дней и ни в одном из них не использовали более 300 импульсов LFR חTMS. P.B. Fitzgerald, J. Benitez, A. de Castella et al. [44] двустороннюю пТМС (1 Гц пТМC справа на DLPFC и с частотой 10 Гц пТMC слева на DLPFC) сравнивали с ложной стимуляцией у 50 пациентов с резистентной депрессией (по 25 пациентов). Лечение продолжалось до 6 недель, дольше, чем в любом ранее опубликованном исследовании. В этой работе отмечено преимущество двусторонней пТМС над фиктивной стимуляцией. Выявлена разница между группами уже в течение 2 недель лече- ния ( $\mathrm{F}$ критерий 1,25 , df $25,5, \mathrm{p} \leq 0,001)$, которые остались значимыми к концу испытания (F 5,44, df 3,9, p=0,005). Пациенты продолжали отвечать на воздействие в течение 6 недель активного лечения. К концу исследования 13 из 25 из пациентов, получающих двустороннюю пТМС (более 50\%) и только 2 в группе с фиктивной пТМС соответствовали критериям ответа на лечение по HDRS. 9 пациентов в группе с двусторонним воздействием (36\%) отвечали критериям клинической ремиссии и таких пациентов без воздействия (фиктивная пТМС) не было. $45 \%$ пациентов в группе с фиктивной пТМC, перешедшие на двустороннее воздействие, в конце испытания отвечали таким же образом - в 33\% отмечены критерии ремиссии. Эти ответы и показатели ремиссии были выше, чем в предыдущих плацебо контролируемых исследованиях и указывали на клинически значимый уровень ответа, особенно учитывая, что это была устойчивая к лечению группа пациентов (среднее число неудачных курсов лечения антидепрессантами 5,9, SD 3,0) с симптомами от умеренной до тяжелой степени.

На сегодняшний день исследования эффективности, оценивающие HFL пTMC, показывают, что, хотя пTMS является эффективной лечебной процедурой, под вопросом остается величина этого клинического эффекта. Все метаанализы литературы по оценке эффективности пТМС включают исследования, которые ограничены несовместимыми способами определения и количественного определения устойчивости к лечению в сравнении с оценкой лекарственного лечения и диагностической неоднородностью. Еще одна проблема - это связь между серьезностью симптомов депрессии и ответом на плацебо. В исследованиях приведены доказательства того, что при сравнении методов лечения депрессии пациенты с тяжелыми депрессивными симптомами имеют более низкие показатели ответа на плацебо (оценка по 17-балльной шкале HDRS 25 или более). Поэтому в работах, включающих в изучение пациентов с легкими и умеренными депрессивными симптомами, частота ответов на плацебо достигает $50 \%$, а это может подорвать преимущества активного лечения пТМС [23].

Существует несколько факторов, лежащих в основе скромной терапевтической эффективности пТМС при лечении депрессии. Большинство этих изучений включало только левостороннюю стимуляцию DLPFC, что является ограничением, если учесть, что при использо- 
вании ЭСТ для лечения резистентной депрессии эффект меньше при односторонней стимуляции, чем двусторонней. Субоптимальные методы использованы при планировании стимуляции DLPFC (5-сантиметровый передний метод, ограничивающий лечебный потенциал изначально целевой формы лечения). Продолжительность лечения была короткой (до 2 недель). Интенсивность стимуляции могла быть недостаточной, потому что не принималось во внимание расстояние от катушки до коры головного мозга, которое имеет особое значение, как параметр для антидепрессивного эффекта пТМС. Возможно, это связано с тем, что все исследования до настоящего времени следуют стандартной процедуре, основанной на ранних исследованиях ТМС, включавшей определение локализации моторного коркового сайта, что приводило при стимуляции в этой зоне к максимальной активации периферической мышцы руки (abductor pollicis brevis) и последующего измерения в 5 см спереди по парасагиттальной линии над поверхностью кожи головы (метод 5 см). Такая методика оценена U. Herwig, F. Padberg, J. Unger et al. [45], авторы локализовали место стимуляции для 5-сантиметрового метода использованием нейронавигационной системы и структурной МРТ. Только у 7 из 22 субъектов метод 5 см соответствовал локализации DLPFC (по Бродману, как BA 9). У 15 пациентов катушка располагалась над более задними областями, в основном над премоторной корой. Результаты показывают, что это исследование могло переоценить даже успех 5сантиметрового метода по определению локализации DLPFC. В системе Бродмана зона BA 9 представляется обширной. Более свежие измерения DLPFC обозначили её как более ограниченную область. Используя координаты для ВА 9 и поле 46, описанные в одном из исследований [46], повторно проанализировано расположение места стимуляции, определенное U. Herwig, F. Padberg, J. Unger et al. с помощью координат Talairach [47]. Результаты показали, что из 22 пациентов только однажды 5сантиметровым методом разместили катушку над ВА 9, и ни в коем случае она не была помещена над ВА 46. В совокупности эти результаты предположили, что традиционные методы для определения места стимуляции DLPFC могут быть неоптимальными и этим можно объяснить скромные терапевтические эффекты пТМС при депрессии в некоторых исследованиях [47].
Два последних терапевтических обсуждения, которые необходимо учитывать, относятся к интенсивности стимуляции и продолжительности лечения (дней стимуляции). В предыдущих исследованиях предполагается, что расстояние от катушки до коры является важным терапевтическим моментом по нескольким причинам. Стимуляция с высокой интенсивностью может способствовать значительному дискомфорту, росту неблагоприятных последствий (головная и лицевая боль), что может привести к прекращению лечения. Чрезмерная стимуляция коры снижает фокальность, обеспечиваемую 8-образной катушкой, что может влиять на результаты лечения. В отличие от интенсивной, низкоинтенсивная стимуляция может привести к вероятному снижению или отсутствию терапевтической эффективности. T.e. регулирование интенсивности стимуляции на основе расстояния от катушки до коры головного мозга является эффективным способом оптимизации лечения пТМС при минимизации нежелательных эффектов [48, 49].

Обсуждается, что сроки от 4 до 6 недель более эффективны, чем короткое лечение. При продолжительности от 4 до 6 недель и более эффективность лечения выше, чем при 2-4недельной терапии. Когда продолжительность лечения увеличивается до 12 недель, хотя и без слепого контроля (Mark Demitrack, Neuronetics Inc, 2007 June 5, Personal Communication), еще до $20 \%$ пациентов достигают терапевтического ответа [48].

Предполагают, что депрессия чаще ассоциируется с гиповозбудимостью над левой DPLFC и повышенной возбудимостью над правой DPLFC. Доказательство в поддержку этого утверждения и причина, почему высокая частота (10 Гц) пТМС была первоначально применена клевой DLPFC и низкочастотная (1 Гц пТМC изначально использована в правой DLPFC для получения антидепрессивного ответа, относится к пациентам с левосторонними инсультами (анатомический эквивалент гиповозбудимости), испытывающим депрессию чаще, чем в общей популяции, и у пациентов с правосторонними инсультами чаще наблюдаются маниакальные симптомы, чем в популяции в целом. Исследования МРТ-изображений показали, что депрессия может включать нарушение регуляции активности коры - более низкая активность в левой DPLFC и более высокая активность в правой DPLFC. 
Кроме того, показано, что эффективность лечения пТМС депрессии связана с нормализацией гиповозбудимости в левой DPLFC и нормализацией гипервозбудимости в правом полушарии, что согласуется с выводом, что пТМС высоких частот повышает возбудимость в коре, а пТМС низких - снижает возбудимость в коре головного мозга. При использовании пТМС частотой 10 Гц в правом DLPFC (равносильно увеличению возбудимости в правом DLPFC) появляются дисфорические симптомы [14]. Прямые доказательства, связывающие изменения в возбудимости в DLPFC на антидепрессивный ответ, отсутствуют. Р.B. Fitzgerald, A. Sritharan, Z.J. Daskalakis et al. [50] и H. Yang [51] продемонстрировали, что у пациентов, получающих пТМС с частотой 1 Гц на правую DLPFC, отмечено двустороннее снижение активности в средней лобной извилине. Напротив, пТMC с частотой 10 Гц на левую DLPFC увеличила активацию в левом прекунеусе и нескольких дополнительных регионах. Ответ на стимуляцию правой DLPFC мTMC с частотой 1 Гц был связан с двусторонним снижением лобной активации.

Изучено, может ли моторная кортикальная возбудимость предсказать ответ на лечение пТМС у 60 пациентов с резистентной депрессией (54 - большое депрессивное расстройство, 6 - биполярная депрессия) [52]. Из них 46 получали антидепрессанты, стабилизаторы настроения и нейролептики. Были выявлены тенденция к снижению возбудимости в покое в левом полушарии, повышенная возбудимость над правой моторной корой, значительная положительная корреляция между повышенной возбудимостью правого полушария и тяжестью симптомов по шкале депрессии Бека и Краткой Психиатрической Рейтинговой Шкале и более низкая возбудимость в левой моторной коре, что предсказывает более слабый ответ на лечение пТМС, особенно у пациентов с преобладанием меланхолических симптомов. В совокупности результаты соответствуют литературе об инсульте, предполагающей, что депрессия связана со снижением возбудимости в левой лобной коре и повышенной возбудимостью в правой лобной коре.

Вышеприведенные выводы ограничены двумя моментами. Во-первых, полученные результаты могут неадекватно отражать нейрофизиологические процессы, опосредующие корковую возбудимость. Скорее всего, они связаны с регионарным мозговым кровотоком. Во- вторых, исследования с использованием ТМС для оценки возбудимости ограничены моторной корой, а область мозга, менее актуальная для феноменологии депрессии по сравнению c DLPFC, изучалась меньше. Имеются технические ограничения, препятствующие способности непосредственно индексировать возбудимость в DLPFC. Это можно достигнуть объединением ТМС с ЭЭГ, что позволяет проводить прямую количественную оценку вызванной кортикальной активации, генерируемой одним импульсом ТМС. Например, одноимпульсная TMC на DLPFC генерирует вызванные потенциалы коры на ЭЭГ, возрастает индекс возбудимости в коре головного мозга [53]. S.K. Esser, R. Huber, M. Massimini et al. [54] продемонстрировали, что высокочастотная пТМС, доставляемая через моторную кору, повышает возбудимость в двигательной коре, так же как с помощью одноимпульсной индуцированной TMC индексируются вызванные в коре потенциалы. Эти выводы соответствуют представлению о механизмах терапевтической эффективности пТМС при лечении резистентной депрессии. Т.е. если лечение HFL пTMC при депрессии связано с повышенной возбудимостью левого DLPFC, а лечение LFR пTMC при депрессии связано с пониженной возбудимостью правого DLPFC, то выяснение этих процессов может оказаться решающим для понимания терапевтических механизмов, вовлеченных в эффективность лечения резистентной депрессии. Будущие исследования, которые объединят ТМС с ЭЭГ для оценки кортикальной возбудимости, могут быть использованы для индексации изменений возбудимости в DLPFC как маркера терапевтического ответа, могут помочь оптимизировать эффекты лечения и выяснить вопросы патофизиологии этого расстройства.

\section{ЗАКЛЮЧЕНИЕ}

пТМС является многообещающей терапией для лечения резистентной депрессии, однако пока ещё скромная клиническая эффективность ограничивает её распространение. Это связано с 4 ключевыми методологическими ограничениями: лечение только с помощью HFL пTMC или одной LFR пTMC, неадекватные методы нацеливания или идентификации DLPFC, ограничение продолжительности лечения (от 2 до 4 недель), неоптимальные методы определения интенсивности лечения. Изучение результатов лечения на основе литературных данных позволило предположить, что применение двусторонней пТМС может улучшить эффективность 
лечения, чем использование односторонней пТМС. Хотя исследований, сравнивающих двустороннюю стимуляцию с односторонней и фиктивной стимуляцией, на материале большого рандомизированного контролируемого исследовании нет. Применение пТМС напрямую на DLPFC через кортикальную MPT может усилить терапевтическую эффективность пТМС в сравнении с применением 5сантиметрового метода. Оптимизировать лечение можно за счёт увеличения его продолжительности (до 6 недель) и повышения интенсивности стимуляции при изменении расстояния от катушки до коры. Эти изменения могут повысить эффективность пТМС для резистентной к лечению депрессии. Важно, чтобы в крупных исследованиях по лечению депрессии были изучены способы терапевтической эффективности для лучшего понимания механизмов лечебного ответа. Оценка возбудимости коры (позитронно-эмиссионной томографией или сочетанием ТМС с ЭЭГ), как потенциальный механизм терапевтического ответа, с помощью этих новых нейрофизиологических подходов может быть решающим и потенциально может изменить фокус приложения пТМС и её применения, улучшая доступные варианты лечения депрессии. Если такие подходы к лечению пТМС не будут реализованы, то риск того, что пТМС продолжит демонстрировать скромную эффективность при лечении депрессии останется, ограничивая её потенциал.

\section{КОНФЛИКТ ИНТЕРЕСОВ}

Авторы заявляют об отсутствии возможных конфликтов интересов в связи с публикацией данной статьи.

\section{ИСТОЧНИК ФИНАНСИРОВАНИЯ}

Авторы декларируют об отсутствии финансирования и спонсорской поддержки при проведении исследования.

\section{ЛИТЕРАТУРА/REFERENCES}

1. Смулевич А.Б. Депрессии в клинической практике врачей общемедицинских специальностей. РМЖ. 2011; 9: 597-99. Smulevich A.B. Depressii v klinicheskoy praktike vrachey obshchemeditsinskikh spetsial'nostey [Depression in clinical practice of doctors of General medical specialties]. Russkiy meditsinskiy zhurnal - Russian Medical Journal. 2011; 9: 597-99 (in Russian)

2. Абрамов А.Н. Роль и место феномена беспомощности в структуре депрессивных расстройств различной этиологии. Национальное здоровье . 2015; 2: 60-5. Abramov A.N. Rol' i mesto fenomena bespomoshchnosti v strukture depressivnykh rasstroystv razlichnoy etiologii [The role and place of the phenomenon of helplessness in the structure of depressive disorders of various etiologies]. Natsional'noye zdorov'ye - National Health. 2015; 2:60-5 (in Russian).

3. Rush A.J., Kraemer H.C., Sackeim H.A. et al. Report by the ACNP Task Force on Response and Remission in Major Depressive Disorder. Neuropsychopharmacology. 2006; 31: 1841-53.

4. Нельсон А.И. Электросудорожная терапия в психиатрии, наркологии и неврологии. 3-е изд. М. : Бином. Лаборатория знаний, 2015: 371. Режим доступа: https://www.books-up.ru/ru/book/ elektrosudorozhnaya-terapiya-v-psihiatrii-narkologiii-nevrologii-3748048/ Nel'son A.I. Elektrosudorozhnaya terapiya $\mathrm{v}$ psikhiatrii, narkologii i nevrologii [Electroconvulsive therapy in psychiatry, narcology and neurology]. Third edition. Moscow: Publishing House Binom. Knowledge laboratory, 2015: 371 (in Russian).

5. Иванов М.В., Петрова Н.Н., Королева А.Е., Чомская В.М., Калева М.А. Электросудорожная терапия: история и современная практика. Психиатрия и психофармакотерапия. 2013; 15 (3): 18-23. Ivanov M.V., Petrova N.N., Koroleva A.Ye., Chomskaya V.M., Kaleva M.A. Elektrosudorozhnaya terapiya: istoriya i sovremennaya praktika [Electroconvulsive therapy: history and modern practice]. Psikhiatriya $i$ psikhofarmakoterapiya. Psychiatry and Psychopharmacotherapy. 2013; 15 (3): 18-23 (in Russian).

6. TeleEMG LLC Neurosoft TMS therapy 510(k) K161519 dated December 22, 2016. Accessed 20 Feb 2018. http://www.fda.gov/MedicalDevices/Device RegulationandGuidance/default.htm

7. Белова А.Н., Балдова С.Н. Транскраниальная магнитная стимуляция: клиническое применение и научные перспективы. Успехи современного естествознания. 2015; 1 (9): 34-42. Belova A.N., Baldova S.N. Transkranial'naya magnitnaya stimulyatsiya: klinicheskoye primeneniye i nauchnyye perspektivy [Transcranial magnetic stimulation: clinical application and scientific prospects]. Uspekhi sovremennogo yestestvoznaniya Successes of Modern Natural Science. 2015; 1 (9): 34-42 (in Russian).

8. Perera T., George M.S., Grammer G., Janicack P.G., Pascual-Leone A., Wirecki T.S. The clinical TMS society consensus review and treatment recommendation for TMS therapy for major depressive disorder. Brain Stimulation. 2016; 9 (3): 33646. DOI: $10.1016 /$ j.brs.2016.03.010

9. Hoffman R.E., Boutros N.N., Hu S. et al. Transcranial magnetic stimulation and auditory hallucinations in schizophrenia. Lancet. 2000; 355 (9209): 1073-75. DOI: https://doi.org/10.1016/S01406736(00)02043-2

10. Lefkovitz Y., Isserles M., Padberg F., Lisaanby S.H., Bystritsky A., Zia G. et al. Efficacy and safety of deep transcranial magnetic stimulation for major depression: a prospective multicenter randomized 
controlled trial. World Psych. 2015; 14 (1): 464-73. DOI: 10.1002/wps.20199

11. Lefaucheur J.P. Neurophysiology of cortical stimulation. Int. Rev. Neurobiol. 2012; 107: 57-85. doi: 10.1016/B978-0-12-404706-8.00005-X

12. Muller P.A., Dhamne S.C., Vahabzadeh-Hagh A.M. et al. Suppression of motor cortical excitability in anesthetized rats by low frequency repetitive transcranial magnetic stimulation. PloS One. 2014; 9 (3): e91065. DOI: 10.1371/journal.pone.0091065

13. Червяков А.В., Пойдашева А.Г., Коржова Ю.Е., Супонева Н.А., Черникова Л.А., Пирадов М.А. Ритмическая транскраниальная магнитная стимуляция в неврологии и психиатрии. Журнал неврологии и психиатрии им. С.С. Корсакова. 2015; 115 (12): 7-18. Chervyakov A.V., Poydasheva A.G., Korzhova Yu.Ye., Suponeva N.A., Chernikova L.A., Piradov M.A. Ritmicheskaya transkranial'naya magnitnaya stimulyatsiya $\mathrm{v}$ nevrologii i psikhiatrii [Rhythmic transcranial magnetic modulation in neurology and psychiatry]. Zhurnal nevrologii i psikhiatrii imeni S.S. Korsakova - Journal of neurology and psychiatry named after S.S. Korsakov. 2015; 115 (12): 7-18 (in Russian).

http://dx.doi.org/10.17116/jnevro20151151127-18

14. Berlim M.T., Van den Eynde F., Daskalakis Z.J. Clinically meaningful efficacy and acceptability of low-frequency repetitive transcranial magnetic stimulation (rTMS) for treating primary major depression: A meta-analysis of randomized, doubleblind and sham-controlled trials. Neuropsychopharmacology. 2013; 38: 543-51. DOI: 10.1038/npp.2012.237

15. Serafini G., Pompili M., Murri M.B., Respiro M., Ghio L., Girardi P. et al. The effects of repetitive transcranial magnetic stimulation on cognitive performance in treatment-resistant depression. A systematic review. Neuropsychobiology. 2015; 71: 125-39. http://dx.doi.org/10.1159/000381351

16. Berlim M.T., Van Den Eynde F., Tovar-Perdomo S., Daskalakis Z.J. Response, remission and dropout rates following high-frequency repetitive transcranial magnetic stimulation (rTMS) for treating major depression: a systematic review and metaanalysis of randomized, double-blind and shamcontrolled trials. Psychol Med. 2014; 44 (2): 225239. DOI: $10.1017 /$ S0033291713000512

17. Chen J., Zhou C., Wu B. et al. Left versus right repetitive transcranial magnetic stimulation in treating major depression: a meta-analysis of randomised controlled trials. Psychiatry Res. 2013; 210 (3): 1260-1264.

18. Fitzgerald P.B., Daskalakis Z.J. The effects of repetitive transcranial magnetic stimulation in the treatment of depression. Expert Review Med Devices. 2011; 8 (1): 85-95.

19. Daskalakis Z.J., Moller B., Christensen B.K. et al. The effects of repetitive transcranial magnetic stimulation on cortical inhibition in healthy human subjects. Exp Brain Res. 2006; 174 (3): 403-412. http://dx.doi.org/10.1007/s00221-006-0472-0

20. Ren Z., Sahir N., Murakami S. et al. Defects in dendrite and spine maturation and synaptogenesis associated with an anxious-depressive-like phenotype of GABAA receptor-deficient mice. Neuropharmacology. 2015; 88: 171-179. DOI: 10.1016/j.neuropharm.2014.07.019

21. Sanacora G., Mason G.F., Rothman D.L. et al. Increased occipital cortex GABA concentrations in depressed patients after therapy with selective serotonin reuptake inhibitors. Am J Psychiatry. 2002; 159 (4): 663-665. https://doi.org/10.1176/appi.ajp.159.4.663

22. George M.S., Wassermann E.M., Williams W.A. et al. Changes in mood and hormone levels after rapid-rate transcranial magnetic stimulation (rTMS) of the prefrontal cortex. J Neuropsychiatry Clin Neurosci. 1996; 8 (2): 172-180. DOI: 10.1176/jnp.8.2.172

23. Voight J., Carpenter L., Leuchter A. A systematic literature review of the clinical efficacy of repetitive transcranial magnetic stimulation (rTMS) in non-treatment resistant patients with major depressive disorder. BMC Psychiatry. 2019; 19: 3-24. https://doi.org/10.1186/s12888-018-1989-z

24. Zhang J., Cui M., Wu Y., Song H. et al. The effect of low frequency repetitive transcranial magnetic stimulation combined with duloxetine in treatment of depression. Zhongguo Shen Jing Jing Shen Ji Bing Za Zhi (Chinese). 2015; 1 (5): 288-92.

25. Richieri R., Jouvenoz D., Verger A. et al. Changes in dorsolateral prefrontal connectivity after rTMS in treatment-resistant depression: a brain perfusion SPECT study. Eur. J. Nucl. Med. Mol. Imaging. 2017; 44: 1051. https://doi.org/10.1007/s00259017-3640-5

26. George M.S., Wassermann E.M., Williams W.A. et al. Daily repetitive transcranial magnetic stimulation (rTMS) improves mood in depression. $\mathrm{Neu}$ roreport. $1995 ; \quad 6 \quad$ (14): 1853-56. https://www.ncbi.nlm.nih.gov/pubmed/8547583

27. Pascual-Leone A., Rubio B., Pallardo F. et al. Rapidrate transcranial magnetic stimulation of left dorsolateral prefrontal cortex in drug-resistant depression. Lancet. $1996 ; \quad 348 \quad$ (9022): 233-237. DOI:https://doi.org/10.1016/S0140-6736(96)01219-6

28. Kang J.I., Lee H., Jhung K. et al. Frontostriatal Connectivity Changes in Major Depressive Disorder after Repetitive Transcranial Magnetic Stimulation: A Randomized Sham-Controlled Study. J. Clin. Psychiatry. 2016; 77 (9): e1137-e1143. http://dx.doi.org/10.4088/JCP.15m10110

29. Xie J., Chen J., Wei Q. Repetitive transcranial magnetic stimulation versus electroconvulsive therapy for major depression: a meta-analysis of stimulus parameter effects. Neurological Res. 2013; 35 (10): 1084-1091.

30. Klein E., Kreinin I., Chistyakov A., Koren D. Therapeutic efficacy of right prefrontal slow repetitive transcranial magnetic stimulation in major depres- 
sion: a double-blind controlled study. Arch Gen Psychiatry. 1999; 56 (4): 315-20. DOI: 10.1001/archpsyc.56.4.315

31. Aguirre I.K., Carretero B., Ibarro O., Kuhalainen J., Martinez J., Ferrer A. et al. Age predicts lowfrequency transcranial magnetic stimulation efficacy in major depression. Jrl Affect Disord. 2011; 130 (3): 466-69. https://doi.org/10.1016/j.jad.2010.10.038

32. Yang H., Xiang H., Qin Q., Huang Y., Yang Z., Zhang M. A randomized controlled trial of right low frequency rTMS combined with escitalopram in treatment of patients with first-episode depression in general hospitals. J Psychiatry Brain Science. 2017; 2 (5): 2. doi:10.20900/jpbs.20170016

33. Berman R.M., Narasimhan M., Sanacora G. et al. A randomized clinical trial of repetitive transcranial magnetic stimulation in the treatment of major depression. Biol Psychiatry. 2000; 47 (4): 332-37. https://doi.org/10.1016/S0006-3223(99)00243-7

34. Loo C., Mitchell P., Sachdev P. et al. Double-blind controlled investigation of transcranial magnetic stimulation for the treatment of resistant major depression. Am J Psychiatry. 1999; 156 (6): 946-48. https://doi.org/10.1176/ajp.156.6.946

35. Kimbrell T.A., Little J.T., Dunn R.T. et al. Frequency dependence of antidepressant response to left prefrontal repetitive transcranial magnetic stimulation (rTMS) as a function of baseline cerebral glucose metabolism. Biol Psychiatry. 1999; 46 (12): $\quad 1603-13 . \quad$ DOI: $10.1016 /$ S00063223(99)00195-X

36. Fitzgerald P.B., Hoy K.E., Anderson R.J., Daskalakis Z.J. A study of the pattern of response to rTMS treatment in depression. Depress Anxiety. 2016; 33: 746-53. DOI: $10.1002 /$ da.22503

37. Avery D.H., Holtzheimer P.E., Fawaz W. et al. A controlled study of repetitive transcranial magnetic stimulation in medication-resistant major depression. Biol Psychiatry. 2006; 59 (2): 187-194. DOI: https://doi.org/10.1016/j.biopsych.2005.07.003

38. O'Reardon J.P., Solvason H.B., Janicak P.G. et al. Efficacy and safety of transcranial magnetic stimulation in the acute treatment of major depression: a multisite randomized controlled trial. Biol Psychiatry. 2007; $62 \quad$ (11): $1208-1216$. http://dx.doi.org/10.1016/j.biopsych.2007.01.018

39. Loo C.K., Mitchell P.B., Croker V.M. et al. Double-blind controlled investigation of bilateral prefrontal transcranial magnetic stimulation for the treatment of resistant major depression. Psychol Med. 2003; 33 (1): 33-40. DOI: 10.1017/S0033291702006839

40. Cohen C.I., Amassian V.E., Akande B. et al. The efficacy and safety of bilateral rTMS in medicationresistant depression. J Clin Psychiatry. 2003; 64 (5): 613-14.

41. Conca A., Di Pauli J., Beraus W. et al. Combining high and low frequencies in rTMS antidepressive treatment: preliminary results. Hum Psychophar- $\begin{array}{llll}\text { macol. } & 2002 ; & 17 & \text { (7): }\end{array}$ https://doi.org/10.1002/hup.422

42. Hausmann A., Kemmler G., Walpoth M. et al. No benefit derived from repetitive transcranial magnetic stimulation in depression: a prospective, single centre, randomised, double blind, sham controlled "add on" trial. J Neurol Neurosurg Psychiatry. 2004; $\quad 75 \quad$ (2): 320-22. https://www.ncbi.nlm.nih.gov/pmc/articles/PMC17 38930/pdf/v075p00320.pdf

43. Rybak M., Bruno R., Turnier L. et al. An attempt to increase the rate and magnitude of the antidepressant effect of transcranial magnetic stimulation (TMS). A pilot study. German Journal of Psychiatry. 2005; 8 (4): 59-65. http:/www.gjpsy.unigoettingen.de

44. Fitzgerald P.B., Benitez J., de Castella A. et al. A randomized, controlled trial of sequential bilateral repetitive transcranial magnetic stimulation for treatment-resistant depression. Am J Psychiatry. 2006; $\quad 163 \quad$ (1): $\quad 88-94$. https://doi.org/10.1176/appi.ajp.163.1.88

45. Herwig U., Padberg F., Unger J. et al. Transcranial magnetic stimulation in therapy studies: examination of the reliability of "standard" coil positioning by neuronavigation. Biol Psychiatry. 2001; 50 (1): $58-61$. https://doi.org/10.1016/S00063223(01)01153-2

46. Rajkowska G., Goldman-Rakic P.S. Cytoarchitectonic definition of prefrontal areas in the normal human cortex: I. Remapping of areas 9 and 46 using quantitative criteria. Cereb Cortex. 1995; 5 (4): 307-322.

47. Daskalakis Z.J., Levinson A.J., Fitzgerald P.B. Repetitive transcranial magnetic stimulation for major depressive disorder: a review. Canadian J Psychiatry. 2008; 53 (9): 555-566. DOI: 10.1177/070674370805300902

48. Thielscher A., Kammer T. Electric field properties of two commercial figure- 8 coils in TMS: calculation of focality and efficiency. Clin Neurophysiol. 2004; $115 \quad$ (7): 1697-1708. DOI: 10.1016/j.clinph.2004.02.019

49. Chen J., Zhou C., Wu B. et al. Left versus right repetitive transcranial magnetic stimulation in treating major depression: a meta-analysis of randomised controlled trials. Psychiatry Res. 2013; 210 (3): 1260-64.

50. Fitzgerald P.B., Sritharan A., Daskalakis Z.J. et al. A functional magnetic resonance imaging study of the effects of low frequency right prefrontal transcranial magnetic stimulation in depression. J Clin Psychopharmacol. 2017; 27 (5): 488-92.

51. Yang H. A randomized, controlled trial of right low frequency rTMC combined with first-episode depression in general hospitals. JPBS. 2017; 2 (5): 2.

52. Fitzgerald P.B., Brown T.L., Marston N.A. et al. Motor cortical excitability and clinical response to rTMS in depression. J Affect Disord. 2004; 82 (1): 71-6. https://doi.org/10.1016/j.jad.2003.09.014 
53. Komssi S., Kahkonen S. The novelty value of the combined use of electroencephalography and transcranial magnetic stimulation for neuroscience research. Brain Res Brain Res Rev. 2006; 52 (1): 183-192. http://dx.doi.org/10.1016/j.brainresrev.2006.01.008

54. Esser S.K., Huber R., Massimini M. et al. A direct demonstration of cortical LTP in humans: a com- bined TMS/EEG study. Brain Research Bulletin. 2006; $69 \quad$ (1): 86-94. https://doi.org/10.1016/j.brainresbull.2005.11.003

Поступила в редакцию 19.10.2018 Утверждена к печати 18.02.2019

Бахмутский Иван Николаевич, аспирант ФГБОУ ВО «Кубанский государственный медицинский университет» МЗ России, врач-психиатр ГБУЗ «Специализированная клиническая психиатрическая больница № 1 » 3 Краснодарского края.

Косенко Виктор Григорьевич, доктор медицинских наук, профессор, заведующий кафедрой психиатрии ФПК и ППС ФГБОУ ВО «Кубанский государственный медицинский университет» МЗ России.

Шулькин Леонид Маркович, кандидат медицинских наук, доцент кафедры психиатрии ФПК и ППС ФГБОУ ВО «Кубанский государственный медицинский университет» МЗ России.

Губа Светлана Александровна, врач-психиатр ГБУЗ «Специализированная клиническая психиатрическая больница № 1» МЗ Краснодарского края.

Бахмутский Николай Георгиевич, доктор медицинских наук, профессор кафедры онкологии ФПК и ППС ФГБОУ ВО «Кубанский государственный медицинский университет» МЗ России.

Бахмутский Николай Георгиевич, bachnik@mail.ru

УДК 616.89-008.454:615.86:159.938.362.33

For citation: Bakhmutsky I.N., Kosenko V.G., Shulkin L.M., Guba S.A., Bakhmutsky N.G. Repeated transcranial magnetic stimulation in the treatment of depression (literature review). Siberian Herald of Psychiatry and Addiction Psychiatry. 2019; 1 (102): 87-98. https://doi.org/10.26617/1810-3111-2019-1(102)-87-98

\title{
Repeated transcranial magnetic stimulation in the treatment of depression (literature review)
}

\author{
Bakhmutsky I.N. ${ }^{1,}{ }^{2}$, Kosenko V.G. ${ }^{1}$, Shulkin L.M. ${ }^{1}$, Guba S.A. ${ }^{2}$, Bakhmutsky N.G. ${ }^{1}$ \\ ${ }^{1}$ Kuban State Medical University \\ Sedin Street 4, 350063, Krasnodar, Russian Federation \\ ${ }^{2}$ Specialized Clinical Psychiatric Hospital no. 1 \\ Krasnaya Street 1, 350000, Krasnodar, Russian Federation
}

\begin{abstract}
Repetitive transcranial magnetic stimulation (rTMS) is an effective treatment for depression. Numerous studies have presented evidence that rTMS is indeed a promising treatment, but its effectiveness is often modest. In this paper, we consider this evidence and discuss several reasons that can explain the modest therapeutic efficacy in most of them, including: the most frequent use of only left-sided stimulation of the dorsolateral prefrontal cortex (DLPFC), which may not be optimal when compared with bilateral stimulation; the use of suboptimal methods for targeting DLPFC (that is, the $5 \mathrm{~cm}$ anterior method used), thereby limiting the potential of therapy only to the target form of treatment a short (from 2 to 4 weeks) duration of therapy and intensity of stimulation during the procedure may not be sufficient if the distance between the coil and the cortex, on which the rTMS-induced antidepressant response is not taken into account. Future research aimed at eliminating the aforementioned limitations is needed to potentially optimize the effectiveness of this promising treatment for depression, especially its pharmacoresistant forms. It is also important that the work is aimed at investigating the mechanisms of the therapeutic efficacy of rTMS, since their understanding can be transformed into successful treatment. For many reasons to be considered, the excitability of the cerebral cortex may be an especially important mechanism related to therapeutic efficacy in treating depression.
\end{abstract}

Keywords: depression, repetitive transcranial magnetic stimulation, cerebral cortex.

Received October 19.2018

Accepted February 18.2019

Bakhmutsky Ivan N., post-graduate student, "Kuban State Medical University" of the Ministry of Health of the Russian Federation; psychiatrist, "Specialized Clinical Psychiatric Hospital no. 1" of the Ministry of Health of the Krasnodar Territory. 
Kosenko Victor G., MD, Prof., Head of the Department of Psychiatry of the Faculty of Advanced Training and Professional Retraining of Specialists "Kuban State Medical University" of the Ministry of Health of the Russian Federation.

Shulkin Leonid M., PhD, Associate Professor of the Department of Psychiatry of the Faculty of Advanced Training and Professional Retraining of Specialists, "Kuban State Medical University" of the Ministry of Health of the Russian Federation.

Guba Svetlana A., psychiatrist, "Specialized Clinical Psychiatric Hospital no. 1" of the Ministry of Health of Krasnodar Territory.

Bakhmutsky Nikolay G., MD, Professor of the Department of Oncology of the Faculty of Advanced Training and Professional Retraining of Specialists, "Kuban State Medical University" of the Ministry of Health of the Russian Federation.

$\triangle$ Bakhmutsky Nikolay G., bachnik@mail.ru 